\section{Bei Patienten mit Leberzirrhose}

\section{Antibiotikum reduziert Rezidivrisiko für hepatische Enzephalopathie}

Die hepatische Enzephalopathie ist eine häufige Komplikation einer Leberzirrhose, die die Lebensqualität deutlich einschränkt und eine hohe Rezidivneigung aufweist, sodass eine Rezidivprophylaxe dringend erforderlich ist. Dazu eignen sich Antibiotika wie Rifaximin, das bei oraler Einnahme praktisch nicht aus dem Darm resorbiert wird und ein breites Wirkspektrum gegen sowohl aerobe als auch anaerobe gram-positive und gram-negative Darmkeime besitzt. Es reduziert deutlich das Rezidivrisiko für eine hepatische Enzephalopathie.

Eine Folge der Leberzirrhose ist die Immobilisierung des Darmtrakts, mit der ein übermäßiges bakterielles Wachstum und eine erhöhte intestinale Permeabilität einhergeht, durch die es wiederum zu vermehrter Translokation von Bakterien und Endotoxämie kommen kann. Tatsächlich treten bakterielle Infektionen bei Patienten mit Leberzirrhose häufig auf und sind mit einer 30-Tages-Mortalität von ca. $30 \%$ und einem weiteren 30\%igen Sterberisiko binnen eines Jahres assoziiert. Die Beeinflussung der Darmflora, von der dieses Infektionsrisiko vor allem ausgeht, sollte deshalb die Prognose bei Leberzirrhose verbessern.

In einer randomisierten, placebokontrollierten Studie [1], so Prof. Dr. Jochen Labenz, Siegen, hatten daher 299 Zirrhose-Patienten, die während der sechs vorangegangenen Monate mindestens zwei Episoden von hepatischer Enzephalopathie erlebt hatten, sechs Monate lang entweder Placebo oder zweimal täglich $550 \mathrm{mg}$ Rifaximin erhalten. Primärer Endpunkt war die Zeit bis zum ersten Rezidiv einer hepatischen Enzephalopathie; das Risiko dafür wurde durch das Antibiotikum hochsignifikant mehr als halbiert mit einer Hazard Ratio von 0,42 (95\%-KI $0,28-0,64 ; \mathrm{p}<0,001)$ und einer «Number-needed-to-treat» (NNT) von 4, d.h. vier Patienten müssen ein halbes Jahr lang Rifaximin erhalten, um eine Episode von hepatischer Enzephalopathie zu verhindern. Das Ausmaß der Risikoreduktion war in allen untersuchten Subgruppen von Patienten vergleichbar. Das Risiko für eine stationäre Aufnahme wegen einer hepatischen Enzephalopathie wurde durch Rifaximin ungefähr genauso stark reduziert (HR 0,50; 95\%KI 0,29-0,87; $p=0,01$ ).

\section{Sicherheit und Lebensqualität: positiv}

Rifaximin war sicher: Es ergaben sich keine Unterschiede bezüglich der Anzahl unerwünschter Ereignisse (80,0\% unter Rifaximin, $79,9 \%$ unter Placebo [1]). Die Lebensqualität wird durch Rifaximin nicht nur nicht beeinträchtigt, sondern sogar verbessert, wie die Auswertung des «Chronic Liver Disease Questionnaire» (CLDQ) ergab, der für 219 Patienten vorlag [2]: Fatigue, abdominelle und systemische Symptome, Aktivität, emotionale Funktion, Besorgnis und Lebensqualität insgesamt verbesserten sich durchweg signifikant.

Wie die Gabe von Laktulose bei der Verhinderung von hepatischen Enzephalopathien im Vergleich zur Gabe von Rifaximin abschneidet, untersuchten US-amerikanische Autoren retrospektiv anhand der Krankenakten von 145 Patienten, die mindestens sechs Monate lang Laktulose und danach mindestens sechs Monate lang Rifaximin (dreimal täglich $400 \mathrm{mg}$ ) erhalten hatten [3]: Die Anzahl der stationären Aufnahmen (0,5 vs. 1,$6 ; \mathrm{p}<0,001)$ während der sechs Monate war unter Rifaximin ebenso reduziert wie die Zahl der im Krankenhaus verbrachten Tage (2,5 vs. 7,3; p < 0,001), die Kosten pro Patient für die stationäre Behandlung (14222 vs. 56635 USD) und die Häufigkeit von Nebenwirkungen wie Diarrhö, Flatulenz oder Schmerzen. Deutlich besser als für Laktulose war unter Rifaximin die Compliance.

\section{Andauernde Wirksamkeit von Rifaximin}

Die Wirkung von Rifaximin ist lang andauernd, wie zwei Studien belegen:

In einer retrospektiven Auswertung der Daten von 203 Patienten mit hepatischer Enzephalopathie [4] zeigte sich, dass nach einem Jahr noch $81 \%$ der Patienten, die Rifaximin als Monotherapie erhalten hatten, in Remission waren, von denen, die das Antibiotikum in Kombination mit Laktulose bekommen hatten, nur $67 \%$.

Um die tatsächliche Langzeit-Wirksamkeit zu testen, wurden 23 Patienten, die auf Rifaximin mit einer Verbesserung der Hämodynamik in der Leber reagiert hatten, in einer Fall-Kontroll-Studie fünf Jahre lang beobachtet [5]. Sie erhielten Rifaximin weiter (1200 $\mathrm{mg} / \mathrm{d}$ ) und wurden mit der doppelten Anzahl von Patienten verglichen, die ihnen bezüglich Alter, Geschlecht und Child-Pugh-Score entsprachen, aber kein Rifaximin bekamen. Rifaximin war mit einer signifikanten Verminderung des Risikos für Varizenblutungen $(35,0 \%$ vs. 59,5\%; $p=0,011$ ), hepatische Enzephalopathie (31,5\% vs. $47,0 \% ; \mathrm{p}=0,034)$, spontane bakterielle Peritonitis $(4,5 \%$ vs. $46,0 \% ; p=0,027)$ und hepatorenales Syndrom (4,5\% vs. $51,0 \%$; $\mathrm{p}=$ $0,037)$ verbunden. Vor allem aber wurde die Sterblichkeit durch die Antibiose signifikant reduziert: Unter Rifaximin waren nach fünf Jahren noch $61 \%$ der Patienten am Leben, von den Kontrollen lediglich 13,5\% ( $\mathrm{p}=0,012)$.

\section{Fazit}

Die hepatische Enzephalopathie ist eine häufige Komplikation der Leberzirrhose mit hoher Rezidivneigung und starker Einschränkung der Lebensqualität, so Prof. Labenz. Nach einer erfolgreichen Behandlung einer Episode ist daher eine Rezidivprophylaxe zwingend angezeigt. Dafür stellt Rifaximin eine neue Therapieoption dar, die durch gute Wirksamkeit und Verträglichkeit ebenso überzeugt wie durch ein Spektrum erwünschter Sekundäreffekte wie der Verbesserung der Hämodynamik und der Senkung des Peritonitis-Risikos. Bei einem ausgezeichneten Sicherheitsprofil wird die Prognose dieser schwerkranken Patienten deutlich verbessert.

\section{Josef Gulden, Grafrath}

\section{Quelle}

Vortrag bei einem Mittagssymposium «Beethoven \& die Leberzirrhose - Hepatische Enzephalopathie: So behandelt man heute» im Rahmen des 119. Kongresses der Deutschen Gesellschaft für Innere Medizin am 8. April 2013 in Wiesbaden, unterstützt von Norgine GmbH, Marburg.

\section{Referenzen}

$1 \quad$ Bass NM et al.: N Engl J Med 2010;362:1071-81

2 Sanyal A et al.: Aliment Pharmacol Ther 2011;34:853-61.

3 Leevy CB, Phillips JA.: Dig Dis Sci 2007;52:737-41.

4 Neff GW et al.: J Clin Gastroenterol 2012;46:168-71.

5 Vlachogiannakos $\mathrm{J}$ et al.: J Gastroenterol Hepatol 2013;28:450-5.

Weitere Informationen bei

Norgine GmbH

Im Schwarzenborn 4

35041 Marburg

info@norgine.de 


\section{News}

Viszeralmedizin 29 | 3 | 13

Ein wertvoller Beitrag zur Patientensicherheit

\section{Das Pulsoxymeter-Display $\mathbf{P o} \mathbf{0}^{\prime \prime)} \mathbf{D i}^{\mathbf{}}$}

Vor dem Hintergrund der stark gestiegenen Anzahl an ambulanten Operationen verweisen DGAI und BDA auf die Mindestanforderungen an den anästhetischen Arbeitsplatz. Um die höchste Priorität der Patientensicherheit zu unterstreichen, werden diese in einer aktuellen Empfehlung zusammengefasst. Als essentieller Mindeststandard an die apparative Ausstattung werden Pulsoxymeter zur Überwachung auch in der Aufwacheinheit empfohlen. Diese sollten vom Standort des Arztes aus jederzeit einsehbar sein.

Diesen Anforderungen in der Praxis gerecht zu werden, war bislang oft schwierig. Das neu konzipierte Verfahren Pulsoxymeter-Display PoDi wird an die vorhandenen Pulsoxymeter angebunden und überträgt die aktuellen Mess- werte drahtlos per WLAN in Echtzeit an beliebige Empfänger im lokalen Netzwerk. PoDi ist modular aufgebaut, frei skalierbar und besteht aus konfigurierbaren Soft- und Hardwarekomponenten. Die einzigartige Systemarchitektur verfügt über zahlreiche Vorteile. Das Verfahren funktioniert mit allen gängigen Pulsoxymetern und ermöglicht den Empfang von Messund Statusmeldungen auf 8 Windows-Endgeräten zugleich. Eine App für Smart-Phone (iOS, Android) wird die Überwachungsmöglichkeit noch zusätzlich abrunden.

Die einfache Implementierung und die intuitive Bedienung sind weitere Gründe für die gute Akzeptanz dieser Lösung. Durch den Einsatz von PoDi zum postoperativen Monitoring kann somit auch im Praxisalltag den Anforderungen an die lückenlose Überwachung umfänglich entsprochen werden. Die Orientierung an dem Stand der Technik sowie den DGAI-Leitlinien tangiert auch zunehmend haftungsrechtliche Aspekte. Die Patienten werden künftig die Einhaltung der Mindeststandards einfordern. PoDi ist ein wichtiger Beitrag zur Erhöhung der Patientensicherheit und zur Sicherung der Behandlungsqualität. PoDi wurde jüngst mit dem Innovationspreis IT der Initiative Mittelstand prämiert.

Weitere Informationen bei

SoMa-Tec UG

Thomas Böhm

An der Priesterkoppel 11a, 13158 Berlin

info@soma-tec.de

\section{Reizdarmsyndrom mit Obstipation:}

\section{Neue Therapieoption Linaclotid}

Das Reizdarmsyndrom (RDS) ist eine weit verbreitete gastrointestinale Funktionsstörung, die für Betroffene mit einer erheblichen Beeinträchtigung der Lebensqualität verbunden sein kann [1]. Rund ein Drittel der Patienten mit Reizdarmsyndrom leidet an dem Obstipationstyp (RDS-O) der Erkrankung. Typische Symptome, über die Betroffene klagen, sind abdominelle Schmerzen und Beschwerden, Blähungen sowie Obstipation. Das therapeutische Spektrum ist begrenzt, die Behandlung führt häufig nicht zur Symptomfreiheit und ein therapeutischer Gold-Standard existiert nicht [1].

Mit Linaclotid (Constella ${ }^{\circledR}$ ) steht seit Mai 2013 eine neue Therapieoption zur Behandlung des mittelschweren bis schweren Reiz- darmsyndroms mit Obstipation bei erwachsenen Frauen und Männern zur Verfügung. Der innovative Guanylatzyklase-C (GC-C)-Rezeptoragonist ist der erste seiner Klasse, der eine gute Wirksamkeit und ein günstiges Verträglichkeitsprofil in der Behandlung des Reizdarmsyndroms mit Obstipation zeigt sowie die Lebensqualität der Patienten nachweislich steigert [3-5].

In 2 randomisierten, doppelblinden, Placebo-kontrollierten, multizentrischen Phase-III-Studien mit über 1600 Studienteilnehmern erreichten die Patienten, die mit Linaclotid behandelt wurden, eine statistisch signifikante und klinisch relevante Verbesserung ihrer abdominellen Schmerzen, Blähungen und Obstipation. Die Wirkung trat innerhalb der ersten Woche ein und hielt über die gesamte Dauer der Studien an [3-5]. Mit dem 1-mal täglich, oral applizierbaren Linaclotid wird das Spektrum der bislang limitierten Therapieoptionen für die Behandlung von Patienten mit Reizdarmsyndrom mit Obstipation erweitert.

\section{Literatur}

1 Layer P et al: Z Gastroenterol 2011;49(2):237-293.

2 Hungin APS et al.: Aliment Pharmacol Ther 2003; 17:643-650.

3 Rao S et al.: Am J Gastroenterol 2012;107(11): $1714-1724$

4 Chey WD et al: Am J Gastroenterol 2012; 107(11):1702-1712.

5 Quigley EM et al.: Aliment Pharmacol Ther 2013; 37:49-61.

Weitere Informationen bei

Almirall Hermal GmbH

Scholtzstraße 3, 21465 Reinbek

WwW.almirall.de

\section{Ticker+++ Ticker+++ Ticker+++ Ticker+++ Ticker+++ Ticker+++ Ticker+++}

Astellas. Aktuelle Auswertungen der QUEPPStudie zeigen, dass gerade durch einen frühen Therapiebeginn neuropathische Schmerzen effektiv behandelt sowie direkte und indirekte $\mathrm{Be}$ handlungskosten verringert werden können. Die nicht-interventionelle QUEPP-Studie ist die derzeit größte Studie mit Capsaicin $8 \%$ kutanes Pflaster (QUTENZA ${ }^{\mathrm{TM}}$ ). Diese NIS mit über 1000 Patienten liefert darüber hinaus erstmals Daten über die Effektivität von QUTENZA ${ }^{\mathrm{TM}}$.

Ketchum Pleon GmbH

Diana Drescher

diana.drescher@ketchumpleon.com
Amgen. Im Rahmen des Gastrointestinal Cancers Symposium 2013 wurden die Ergebnisse der PAVES-Studie vorgestellt, die den guten Schutzeffekt von Neulasta ${ }^{\circledR}$ vor febrilen Neutropenien erneut bestätigten. Für Patienten, die leitliniengemäß wegen einer myelotoxischen Chemotherapie einer G-CSF-Prophylaxe bedürfen, stellt Pegfilgrastim, das nur 1-mal pro Zyklus gegeben werden muss, eine besonders wirksame Option dar.

Amgen $\mathrm{GmbH}$

Fulvia Kipper

fulvia.kipper@amgen.com
Falk. Chronisch entzündlichen Darmerkrankungen liegt eine intestinale Barrierestörung zugrunde. Als vielversprechende Strategie gegen diesen Defekt gelten die Eier des Schweinepeitschenwurms, mit denen in Pilotstudien bei Morbus Crohn hohe Remissionsraten erreicht wurden. Die Verbesserung der Barrierefunktion mit Phosphatidylcholin (Lecithin) könnte bei Colitis ulcerosa einen Therapiefortschritt bedeuten.

eickhoff kommunikation $\mathrm{GmbH}$

Dr. Maria Ruppert

ruppert@medizinmarketingservice.de 


\section{News}

Viszeralmedizin 29 | 3 | 13

Neue 3D-Technologie erleichtert laparoskopische Eingriffe

Schneller, präziser, patientenschonender

Ende Juni 2013 wird die Firma Olympus das neue Videosystem EVIS EXERA III mit der Videooptik ENDOEYE FLEX 3D auf den Markt bringen. Es erzeugt ein hochauflösendes dreidimensionales Bild des Operationsgebiets mit einer sehr realistischen räumlichen Tiefe, da wie beim menschlichen Auge die Daten von zwei Optiksystemen zusammengeführt werden. Die Besonderheit des Systems ist die in vier Richtungen abwinkelbare Spitze des Instruments, die eine Veränderung der Blickrichtung und somit im Gegensatz zu anderen 3D-Laparoskopen eine Betrachtung aus verschiedenen Winkeln erlaubt.

Erstmals hatten Operateure auf dem Chirurgenkongress in München die Gelegenheit, das neue Laparoskopsystem am Stand des Herstellers auszuprobieren und direkt zu vergleichen, welche Vorteile diese Art der 3D-Darstellung im Vergleich zur 2D-Laparoskopie bringt. Produktmanagerin Meike Hein erläuterte auf dem Kongress, wie die Anwender von dem neuen 3D-System profitieren.

Das räumliche Sehen beruht darauf, dass das rechte Auge und das linke Auge zwei unterschiedliche Bilder aus jeweils leicht versetzten Blickwinkeln liefern, aus denen das Gehirn ein dreidimensionales Bild erstellt. Nur dies erlaubt, Entfernungen und die Größe von Objekten abzuschätzen. Auch für die Endoskopie gilt, dass man für eine 3D-Darstellung zwei Bilder aus zwei verschiedenen Blickwinkeln benötigt.

\section{Bessere räumliche Darstellung} durch zwei optische Systeme

Um dies zu erreichen, gibt es zwei Möglichkeiten:

1. Die Split-Linse: Es wird ein Bild aufgenommen und auf zwei Bildsensoren aufgeteilt. Aus beiden Datensätzen wird das 3D-Bild errechnet.

2. Verwendung von zwei Linsen: Es werden wie beim menschlichen Auge zwei Bilder erzeugt und je einem Bildsensor zugeleitet. Aus den beiden Originalbildern wird das 3D-Bild erstellt.

Der Abstand zwischen den beiden optischen Systemen (Parallaxe) ist bei der Splitlinse kleiner als bei zwei einzelnen optischen Systemen. Je größer er ist, desto besser ist die räumliche Wahrnehmung, je kleiner, desto flacher das 3D-Bild. Split-Linsen-Systeme erzeugen deshalb flachere 3D-Bilder als Systeme mit zwei separaten optischen Systemen.

\section{Dreidimensionales Sehen mit großer Bewegungsfreiheit}

Das neue ENDOEYE FLEX 3D ist mit einem dualen Linsensystem und zwei hochauflösenden Chips ausgestattet. Das gesamte optische System befindet sich in der Spitze des Endoskops («Chip-on-the-Tip»). Diesen Vorteil bietet im Moment kein anderer Hersteller an. Beide Bildsignale werden von einem speziellen Videosystem verarbeitet und zu einem hochauflösenden 3D-Bild zusammengeführt, das der Anwender mit Hilfe einer polarisierenden 3D-Brille auf dem 3D-Monitor betrachten kann.

Das System baut auf bestehenden 2D-Systemen auf und ist mit diesen kompatibel. Es gibt zudem die Möglichkeit, per Knopfdruck von 3D auf 2D umzustellen. Das Gerät ist somit ohne Umbau sofort einsatzbereit. Außerdem ist das 3D-Endoskop einhändig manövrierbar und muss nicht am Tisch fixiert werden. Die Kompatibilität mit herkömmlichen 2D-Systemen bedeutet für den Anwender nicht nur ein hohes Maß an Flexibilität, sondern ist auch im Klinikalltag ökonomisch sinnvoll.

\section{Bessere Übersicht durch abwinkel- bare Spitze}

Ein weiterer großer Vorteil liegt darin, dass die Spitze des neuen 3D-Laparoskops in alle vier Richtungen um bis zu $100^{\circ}$ abgewinkelt werden kann (Abb. 1). Das Operationsfeld lässt sich deshalb nicht nur wie mit herkömmlichen Laparoskopen frontal betrachten, sondern auch aus anderen Blickwinkeln, eventuell sogar «von hinten». Durch die abwinkelbare

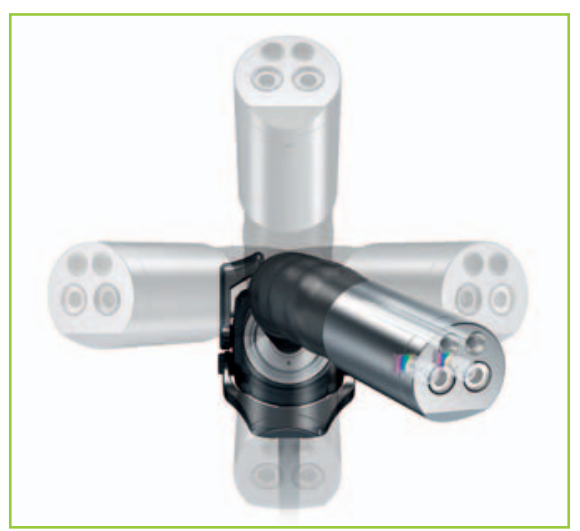

Abb. 1. Detailansicht der Endoskopspitze.

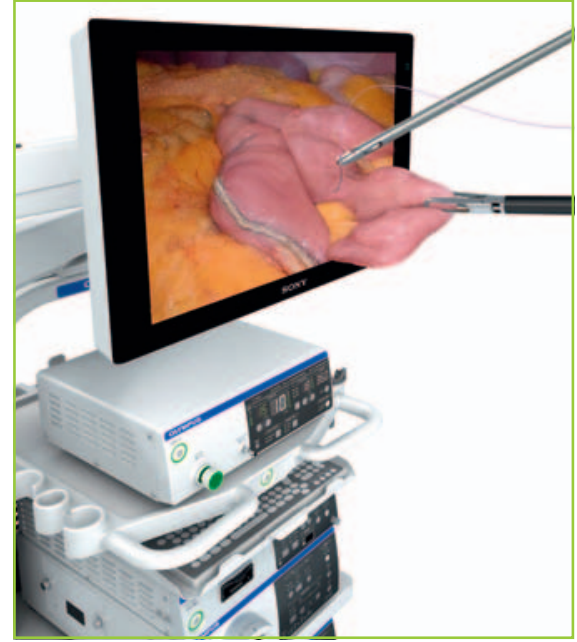

Abb. 2. Die 3D-Ansicht vereinfacht die Beurteilung von Abständen anatomischer Strukturen zueinander und zu den Instrumenten.

Spitze kann das ENDOEYE FLEX 3D unter anderem auch dieselbe Blickrichtung wie ein 2D-30-Endoskop erzeugen. Bei 3D-Endoskopen mit nicht abwinkelbarer Spitze ist dagegen nur eine $0^{\circ}$-Darstellung möglich.

Die durch das duale Linsensystem exaktere räumliche Darstellung hat für den Operateur große Vorteile. Die Hand-Augen-Koordination verbessert sich, weil die Abstände anatomischer Strukturen zueinander und zu den Instrumenten besser zu beurteilen sind (Abb. 2). Die Arbeit am Gewebe, z. B. das korrekte Ausrichten der Nadel beim Nähen und das Wiederaufgreifen des Fadens, fällt leichter und geht auch schneller; Ermüdungserscheinungen bleiben aus. Der Einsatz der 3D-Laparoskopie bietet sich bei Prozeduren an, bei denen viel genäht werden muss oder eine feine Präparation erforderlich ist, z. B. wenn man in der Nähe von Nerven oder Gefäßen arbeitet.

Durch die räumliche Darstellung im Abdomen und die bessere Orientierung des Operateurs kann das Endoskop weiter weg vom Operationsfeld bleiben und muss so seltener gereinigt werden. Ein übermäßiges Bewegen oder Repositionieren der Optik entfällt, das Manipulieren und Operieren wird exakter.

Dr. med. Angelika Bischoff, Planegg

\section{Quelle}

Präsentation anlässlich des 130. Kongresses der Deutschen Gesellschaft für Chirurgie, München, 2. Mai 2013.

Weitere Informationen bei Olympus Deutschland GmbH Produktmanagement Imaging Meike Hein

Wendenstr. 14-18, 20097 Hamburg meike.hein@olympus.de

\section{KARGER}

Fax +497614520714 Information@Karger.co www.karger.com 


\section{News}

Viszeralmedizin 29 | 3 | 13

Chronische Obstipation - Neue Leitlinie setzt Standards

Obstipation: Eine ernst zu nehmende Erkrankung

Die chronische Obstipation ist eine verbreitete gastrointestinale Funktionsstörung, die sich in einer Vielzahl von lästigen Symptomen äußern kann, sagte Frau Dr. Viola Andresen vom Israelitischen Krankenhaus in Hamburg bei einem Satellitensymposium der Firma Shire während des diesjährigen Kongresses der Deutschen Gesellschaft für Innere Medizin (DGIM) in Wiesbaden. Diese zum Teil sehr beeinträchtigende Erkrankung wird durch unterschiedliche primäre oder sekundäre Störungen des Darms und/oder des Beckenbodenbereiches ausgelöst. Die sogenannten ROM-III-Kriterien definieren, wann eine chronische Verstopfung vorliegt. In jedem Fall ist eine sorgfältige Diagnostik zu fordern, um die genaue Genese der Obstipation zu erkennen, so Andresen. Nur dann kann eine sinnvolle Therapie erfolgen.

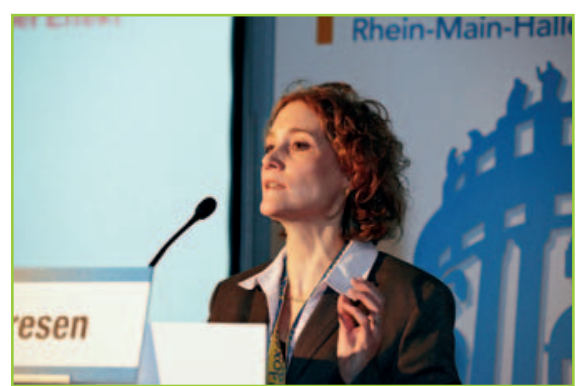

Abb. 1. Dr. Viola Andresen, Israelitisches Krankenhaus Hamburg.

Pathophysiologie der chronischen Obstipation

Prof. Dr. Michael Schemann, Ordinarius für Humanbiologie an der Technischen Universität München, beleuchtete in seinem Vor- trag interessante pathophysiologische Aspekte der chronischen Obstipation. So ist bei einer Gruppe chronisch verstopfter Patienten die Kolontransitzeit verlängert. Man spricht hier von der «Slow Transit Constipation». Dabei sind Frequenz und Amplitude der Kontraktionen der Darmmuskulatur verringert. Es wird ein Zusammenhang vermutet mit einer moderaten Hypoganglionose im Plexus myentericus, verringerter ICC (Interstitielle Cajal-Zellen)-Zelldichte und einer geringen Expression von Strukturproteinen des kontraktilen Apparates. Hierdurch könnten nervale Reflexe des enterischen Nervensystems beeinträchtigt sein. Neue therapeutische Konzepte fokussieren daher auf die Normalisierung der neuromuskulären Übertragung und Motilität. So fördert die Aktivierung der 5-HT4-Rezeptoren durch das Prokinetikum Prucaloprid $\left(\right.$ Resolor $^{\circledR}$ ) die synaptische Verschaltung im enterischen Nervensystem und stimuliert dadurch den peristaltischen Reflex als Grundlage propulsiver Motilität.

Neue interdisziplinäre Leitlinie

Prokinetika haben auch ihren Stellenwert in der neuen S2k-Leitlinie zur «Epidemiologie, Pathophysiologie, Diagnostik und Therapie der chronischen Obstipation», in der sich DGNM (Deutsche Gesellschaft für Gastroenterologie und Motilität) und DGVS (Deutsche Gesellschaft für Verdauungs- und Stoffwechselerkrankungen) gemeinsam auf Grundsätze zum Umgang mit chronischer Obstipation festgelegt haben. Abgesehen von den spezifischen Maßnahmen bei Stuhlentleerungsstörungen, die noch durch defäkationsstimulierende Suppositorien und Klysmen unterstützt werden können, beruht die Therapie der sonstigen chronischen Obstipation auf einem Stufenkonzept, das in $\mathrm{Ab}$ hängigkeit von der Ausprägung der Symptomatik, dem jeweiligen Ansprechen und auch nicht zuletzt der Verträglichkeit und der resultierenden Lebensqualität umgesetzt wird. Bei den Patienten, bei denen Laxantien nicht oder nur unzureichend effektiv sind oder schlecht vertragen werden, stellt Prucaloprid eine gute neue Therapieoption dar. In der Mehrzahl der Fälle wird eine erhebliche Verbesserung von Stuhlfrequenz und Symptomen der Obstipation erreicht.

\section{Dr. Eva Husen-Weiss, Berlin}

\section{Quelle}

Satellitensymposium der Firma Shire Deutschland GmbH anlässlich des 119. Kongresses der Deutschen Gesellschaft für Innere Medizin e.V. (DGIM), 8.4.2013 in Wiesbaden

Weitere Informationen bei

Media Concept GmbH (GPRA)

Kommunikation im Gesundheitswesen

Dr. Peter Gerlach

Friedrich-Ebert-Straße 51, 47179 Duisburg Tel. +49 203 45694-20, Fax -24

pg@mediaconcept.de

\section{Ticker+++ Ticker+++ Ticker+++ Ticker+++ Ticker+++ Ticker+++ Ticker+++}

Dürr Technik. Medizinische Instrumente und OP-Besteck sind vielfach nicht vorschriftsmäBig gereinigt. Eine effiziente und fachgemäße Lösung für die Trocknung von Endoskopen ist das Silent Air System (SAS-38). Die anschlussfertige Kompressoreinheit befreit alle zugänglichen Kanäle der Instrumente von Restfeuchtigkeit. Damit kommen Ärzte einer Empfehlung des Robert-Koch-Instituts nach, das ganz explizit die Verwendung von ölfreier Druckluft bei der Aufbereitung von Endoskopen vorsieht.

Dürr Technik

Elke Sieber

sieber.e@duerr-technik.de
Sanofi. Die Europäische Kommission (EMA) hat Zaltrap ${ }^{\circledR}$ (Aflibercept) in Kombination mit einer Irinotecan/5-Fluorouracil/Folinsäure(FOLFIRI)-Chemotherapie die Zulassung zur Behandlung erwachsener Patienten mit metastasiertem kolorektalem Karzinom (mCRC), die auf ein oxaliplatinhaltiges Therapieregime nicht angesprochen haben oder bei denen es danach zu einer Krankheitsprogression kam, in der Europäischen Union erteilt. Es ist seit März 2013 verfügbar

Sanofi-Aventis Deutschland GmbH

Cristina lannazzo

presse@sanofi.com
MSD SHARP \& DOHME. Eine chronische Infektion mit dem Hepatitis-C-Virus (HCV) schränkt die Arbeitsfähigkeit und Lebensqualität der Betroffenen stark ein. Patienten mit chronischem HCV sollten daher so rasch wie möglich diagnostiziert und behandelt werden. Durch die Entwicklung neuer Medikamente, die das Hepatitisvirus direkt angreifen, sogenannte Proteasehemmer (wie z. B. Boceprevir), sind die Heilungschancen deutlich gestiegen.

MSD SHARP \& DOHME GMBH infocenter@msd.de www.msd.de 